\title{
Repurposing Neuroactive Drugs in Oncology: A Mini-Review
}

ISSN: 2576-9170

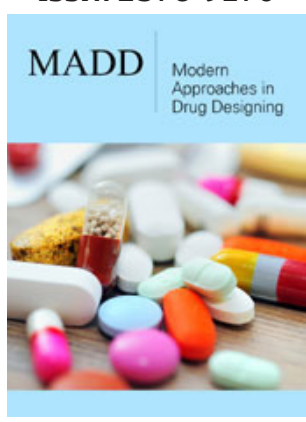

*Corresponding author: Tyler Bland, Department of Pharmaceutical Sciences, College of Pharmacy and Pharmaceutical Sciences, Washington State University, Spokane, WA 99202, USA

Submission: 海 February 28, 2020

Published: March 09, 2020

Volume 2 - Issue 5

How to cite this article: Tyler Bland, Boyang Jason $\mathrm{Wu}$. Repurposing Neuroactive Drugs in Oncology: A Mini-Review. Mod Appro Drug Des.2(5). MADD.000550.2020. DOI: 10.31031/MADD.2020.02.000550.

Copyright@ Tyler Bland. This article is distributed under the terms of the Creative Commons Attribution 4.0 International License, which permits unrestricted use and redistribution provided that the original author and source are credited.

\author{
Tyler Bland* and Boyang Jason Wu
}

Department of Pharmaceutical Sciences, College of Pharmacy and Pharmaceutical Sciences, Washington State University, Spokane, WA 99202, USA

\begin{abstract}
There are many effective treatments on the market in oncology, yet cancer remains the second leading cause of death in the U.S. This is largely due to cancer evolution and the development of drug resistance. One classification of difficult-to-treat cancers is neuroendocrine tumors, which show remarkable similarities with neuronal cells. Mechanisms of disease progression and biomarkers are even shared between neuroendocrine tumors and neurological disorders, providing a strong rationale for repurposing neuroactive compounds, used in the clinic to treat neurological disorders as therapeutics in oncology. Current lines of evidence already support the repurposing of multiple neuroactive drugs in many types of cancer, yet the advantages do not extend to all cancer types, with some neuroactive drugs even showing pro-tumor growth effects. The development of new cancer therapeutics through repurposing drugs that treat other indications holds immense significance for increasing the value of these drugs, along with providing crucial therapies to prolong survival in cancer patients. In the present mini review, we will highlight some of the recent advances in the field of drug repurposing in oncology particularly with respect to neuroactive drugs.
\end{abstract}

Keywords: Cancer; Neuroendocrine tumor; Neurological disorders; Analgesics; Drug repurposing

Abbreviations: Neuroendocrine Tumor (NET), Serotonin (5HT), Alzheimer's Disease (AD), Parkinson's Disease (PD), Selective Serotonin Reuptake Inhibitors (SSRIs), Tricyclic Antidepressants (TCAs), Monoamine Oxidase Inhibitors (MAOIs)

\section{Introduction}

Cancer is the second leading cause of death in the U.S., leading to the loss of over half a million lives in 2019 alone [1]. While there are many available treatments, one hallmark of lethal forms of cancer is the development of drug resistance. Drug resistance gives rise to an evolutionary arms race between cancer and pharmaceutical companies, which spend billions of dollars each year in research and development attempting to discover new and powerful therapies to treat drug-resistant forms of cancer. One main weakness of developing novel first-in-human therapies in oncology is the high failure rate in clinical trials, with success rates remaining less than $4 \%$ for these types of compounds [2]. This low success rate is due to two main factors: 1 . lack of efficacy in the target disease, and/or 2. intolerable toxicity. A more promising approach is drug repurposing or repositioning, which is a strategy for enhancing the value of a drug already used in the clinic for other indications by utilizing it to target diseases other than those it was originally intended to treat. Repurposed drugs, which have a known mechanism of action and toxicological profile, provide a much stronger approach to treat cancers with similar biomarkers and disease mechanisms, due to the probability of higher success rates in clinical trials.

One area of oncology that may greatly benefit from repurposed drugs is the treatment of neuroendocrine tumors (NETs). This difficult-to-treat cancer subtype can arise de novo or can be treatment induced. NETs have both neuronal and endocrine cell properties, expressing neuronal genes and exhibiting a neuronal morphology, while also expressing both neurotransmitter receptors and neurotransmitters such as serotonin (5-HT), neuropeptides, and acetylcholine [3-5]. This large shift in cellular characteristics commonly renders them resistant to current cancer therapies. The study of NET biology is an emerging field of cancer research and is vitally important for the development of novel therapeutics. However, the knowledge of neuronal characteristics in NETs provides a unique opportunity for repurposing neuroactive therapies currently used to treat the multitude of neurological disorders ranging 
from neurodegenerative diseases to pain management. The mini review will focus on select examples of these neuroactive drugs that show promise in repurposing as cancer therapeutics.

\section{Neurological Disorders}

Neurological disorders comprise a wide variety of disorders and diseases ranging from neurodegenerative and mental disorders that can affect mood, thinking, and behavior such as Alzheimer's Disease (AD), Parkinson's Disease (PD), depression, and Schizophrenia, to peripheral nervous system disorders such as neurogenic bladder and neurologic bowel disorder.

One promising area for drug repurposing is in mental disorder treatments. Many biomarkers and disease mechanisms seen in mental disorders are also observed in NETs. Depression has been linked to defects in serotonergic (5-HT) signaling leading to treating patients with antidepressant compounds that increase 5-HT tone such as selective serotonin reuptake inhibitors (SSRIs), tricyclic antidepressants (TCAs), and monoamine oxidase inhibitors (MAOIs). TCA and SSRI treatments have been observed to reduce the incidence of colorectal cancer [6], and MAOIs have demonstrated efficacy in reducing prostate cancer bone metastasis and prolonging survival in mouse models [7]. Conversely, antidepressants have also shown pro-tumorigenic affects. The SSRI fluoxetine (Prozac) has been found to increase the number of brain metastasis in breast cancer due to changes in blood-brain barrier permeability, pro-inflammation, and glial activation in the brain [8].

While 5-HT is one of the main neurotransmitters involved in depression, another biogenic amine neurotransmitter, dopamine, plays a crucial role in PD. Treatments for PD include L-Dopa and carbidopa which are agonists for dopamine receptors and act to overcome the loss of dopaminergic neuronal signaling in PD patients. Dopamine receptor expression and signaling has been observed in NETs and suggests a therapeutic approach to modulate dopaminergic signaling in these tumors [9]. Carbidopa has shown promise suppressing pancreatic cancer growth in preclinical studies [10], while no effect was seen in breast cancer and melanoma [11].

Another area of neuroactive drugs that show promise as NET therapies are those used to treat psychiatric disorders, with multiple antipsychotics showing anti-tumor effects in preclinical studies. With epidemiological studies showing an inverse relationship between schizophrenia treatments and cancer incidence, many studies have investigated the potential of these compounds as primary cancer treatments [12]. Men undergoing long-term treatment with the schizophrenia drug haloperidol have a reduced risk of developing prostate cancer with in vitro evidence suggesting haloperidol may reduce prostate cancer cell growth [13]. The schizophrenic and major depressive disorder drug aripiprazole (Abilify) reduces cell proliferation and tumor growth of glioma, gastric cancer, and colon cancer [14]. Another schizophrenic drug, sertindole, also shows promise in treating breast and gastric cancers $[15,16]$.
Even compounds that do not have psychological activities but affect peripheral nervous system disorders have shown promise for treating cancer. Overactive parasympathetic signaling is implicated in neurogenic bladder and neurologic bowel disorder, leading to the prescription and treatment with anticholinergics $[17,18]$. These therapies have demonstrated efficacy in treating a variety of cancers including lung, colon, bladder, and prostate cancers $[5,19,20]$.

\section{Analgesics}

An unfortunate symptom of many cancers is the incidence of chronic pain, either due to the cancer itself or as a side effect of many cancer therapies. Many patients are prescribed analgesics, or antinociceptive medications, to prevent or dampen the nociceptive signal from the brain. Many of these therapies act directly on peripheral and central nervous systems. While they show strong efficacy modulating pain sensation, they also may be useful in treating tumors directly. Analgesics fall into two categories: 1. opioids, and 2. non-opioids.

The first, and probably most well-known class of analgesics, is opioids. These drugs treat pain sensation by blocking neuronal signaling involved in nociception through activating opioid receptors. The anti-tumor effects of opioids are controversial [21], though some studies have reported the ability of opioids to decrease tumor growth. For instance, morphine treatment was found capable of suppressing lung cancer cell proliferation [22], while no effect was seen in breast tumor growth [23], suggesting that morphine treatment may be beneficial in a cancer type-specific manner.

Non-opioid analgesics are also used to treat pain and have a mechanism of action other than regulating opioid receptor activity. One example is lidocaine, which is commonly used as a local anesthetic to treat dermal and oral pain and has been shown to prevent breast cancer progression and metastasis [24-26], prevent the progression of retinoblastoma [27], and induce cell cycle arrest in colon cancer [28]. The analgesics metamizole and paracetamol have been shown to inhibit pancreatic cancer growth [29] while metamizole and acetaminophen exert cytotoxic effects in colon cancer [30]. Synthetic cannabinoids such as nabilone and cannabidiol also show promise as cancer therapeutics [23], yet this remains controversial [31,32].

\section{Conclusion and Future Perspective}

Overall, neuroactive drugs used in the clinic to treat neurological disorders and pain management represent a promising class of drugs for repurposing as cancer therapeutics. NETs show a remarkable similarity of genotype and phenotype with neurons as directly evidenced by common biomarkers seen in both cell types. This provides strong evidence that neurotherapies will show efficacy in these cancer types. While some evidence already exists in this arena, much more still needs to be explored about these difficult-to-treat cancers, particularly cancer type-specific responses to individual neurotherapy given opposite treatment 
outcomes in different types of cancer for some neurotherapies. Precision medicine may be a potential approach to determine the type of therapy tailored for individual patients by examining the biomarkers expressed in a patient's tumor for prescribing the corresponding treatment. The repurposing nature of these therapies will provide a quick translational timeframe due to the already known mechanism of action and toxicology. We anticipate that many of these neuroactive drugs will enter the oncology market in the near future and will provide treatments to extend the survival of many patients who suffer from difficult-to-treat cancers with limited viable therapeutic options.

\section{Acknowledgement}

Tyler Bland and Boyang Jason Wu contributed to writing the manuscript, which was funded by the Washington State University Office of Commercialization GAP Fund.

\section{Conflict of Interest}

None.

\section{References}

1. Siegel RL, Miller KD, Jemal A (2019) Cancer statistics, 2019. CA Cancer J Clin 69(1): 7-34.

2. Wong CH, Siah KW, Lo AW (2019) Estimation of clinical trial success rates and related parameters. Biostatistics 20(2): 273-286.

3. Pelosi G, Volante M, Papotti M, Sonzogni A, Masullo M, et al. (2006) Peptide receptors in neuroendocrine tumors of the lung as potential tools for radionuclide diagnosis and therapy. Q J Nucl Med Mol Imaging 50(4): 272-287.

4. Patel P, Galoian K (2018) Molecular challenges of neuroendocrine tumors. Oncology Letters 15(3): 2715-2725.

5. Wang N, Yao M, Xu J, Quan Y, Zhang K, et al. (2015) Autocrine activation of CHRM3 promotes prostate cancer growth and castration resistance via CaM/CaMKK-mediated phosphorylation of Akt. Clin Cancer Res 21(20): 4676-4685.

6. Chubak J, Boudreau DM, Rulyak SJ, Mandelson MT (2011) Colorectal cancer risk in relation to antidepressant medication use. Int J Cancer 128(1): 227-232.

7. Wu JB, Yin L, Shi C, Li Q, Duan P, et al. (2017) MAOA-dependent activation of Shh-IL6-RANKL signaling network promotes prostate cancer metastasis by engaging tumor-stromal cell interactions. Cancer Cell 31(3): 368-382.

8. Shapovalov Y, Zettel M, Spielman SC, Amico-Ruvio SA, Kelly EA, et al. (2014) Fluoxetine modulates breast cancer metastasis to the brain in a murine model. BMC Cancer 14: 598.

9. Srirajaskanthan R, Watkins J, Marelli L, Khan K, Caplin ME (2009) Expression of somatostatin and dopamine 2 receptors in neuroendocrine tumours and the potential role for new biotherapies. Neuroendocrinology 89(3): 308-314.

10. Ogura J, Miyauchi S, Shimono K, Yang S, Gonchigar S, et al. (2017) Carbidopa is an activator of aryl hydrocarbon receptor with potential for cancer therapy. Biochem J 474(20): 3391-3402.

11. Duarte D, Amaro F, Silva I, Silva D, Fresco P, et al. (2019) Carbidopa alters tryptophan metabolism in breast cancer and melanoma cells leading to the formation of indole-3-acetonitrile, a pro-proliferative metabolite. Biomolecules 9(9): 409.
12. Tabarés-Seisdedos R, Rubenstein JL (2013) Inverse cancer comorbidity: a serendipitous opportunity to gain insight into CNS disorders. Nat Rev Neurosci 14(4): 293-304.

13. Friedman GD, Habel LA, Achacoso N, Sanders CM, Oyer HM, et al. (2020) Haloperidol and prostate cancer prevention: More epidemiologic research needed. Perm J 24.

14. Kim MS, Yoo BC, Yang WS, Han SY, Jeong D, et al. (2018) Src is the primary target of aripiprazole, an atypical antipsychotic drug, in its anti-tumor action. Oncotarget 9(5): 5979-5992.

15. Zhang W, Zhang C, Liu F, Mao Y, Xu W, et al. (2018) Antiproliferative activities of the second-generation antipsychotic drug sertindole against breast cancers with a potential application for treatment of breast-tobrain metastases. Sci Rep 8(1): 1-13.

16. Dai C, Liu P, Wang X, Yin Y, Jin W, et al. (2020) The antipsychotic agent sertindole exhibited antiproliferative activities by inhibiting the STAT3 signaling pathway in human gastric cancer cells. J Cancer 11(4): 849857.

17. Dorsher PT, McIntosh PM (2012) Neurogenic bladder. Advances in Urology, Article ID: 816274.

18. Padhy SK, Sahoo S, Mahajan S, Sinha SK (2015) Irritable bowel syndrome: Is it "irritable brain" or "irritable bowel"? J Neurosci in Rural Pract 6(4): 568-577.

19. Löfling L, Sundström A, Kieler H, Bahmanyar S, Linder M (2019) Exposure to antimuscarinic medications for treatment of overactive bladder and risk of lung cancer and colon cancer. Clin Epidemiol 11: 133-143.

20. Kaye JA, Margulis AV, Fortuny J, McQuay LJ, Plana E, et al. (2017) Cancer incidence after initiation of antimuscarinic medications for overactive bladder in the United Kingdom: Evidence for protopathic bias. Pharmacotherapy 37(6): 673-683.

21. Afsharimani B, Cabot P, Parat MO (2011) Morphine and tumor growth and metastasis. Cancer and Metastasis Reviews 30(2): 225-238.

22. Kim JY, Ahn HJ, Kim JK, Kim J, Lee SH, et al. (2016) Morphine suppresses lung cancer cell proliferation through the interaction with opioid growth factor receptor: An in vitro and human lung tissue study. Anesth Analg 123(6): 1429-1436.

23. André Barrière D, Midavaine É, Doré-Savard L, Kirby K, Tremblay L, et al. (2019) Dichotomic effects of clinically used drugs on tumor growth, bone remodeling and pain management. Sci Rep 9: 20155.

24. Wall TP, Crowley PD, Sherwin A, Foley AG, Buggy DJ (2019) Effects of lidocaine and src inhibition on metastasis in a murine model of breast cancer surgery. Cancers (Basel) 11(10): E1414.

25. Freeman J, Crowley PD, Foley AG, Gallagher HC, Iwasaki M, et al. (2019) Effect of perioperative lidocaine, propofol and steroids on pulmonary metastasis in a murine model of breast cancer surgery. Cancers (Basel) 11(5): E613.

26. Johnson MZ, Crowley PD, Foley AG, Xue C, Connolly C, et al. (2018) Effect of perioperative lidocaine on metastasis after sevoflurane or ketaminexylazine anaesthesia for breast tumour resection in a murine model. $\mathrm{Br}$ J Anaesth 121(1): 76-85.

27. Xia W, Wang L, Yu D, Mu X, Zhou X (2019) Lidocaine inhibits the progression of retinoblastoma in vitro and in vivo by modulating the miR-520a-3p/EGFR axis. Mol Med Rep 20(2): 1333-1342.

28. Bundscherer AC, Malsy M, Bitzinger DI, Wiese CHR, Gruber MA, et al. (2017) Effects of lidocaine on HT-29 and SW480 colon cancer cells in vitro. Anticancer Res 37(4): 1941-1945.

29. Malsy M, Graf B, Bundscherer A (2017) Effects of metamizole, MAA, and paracetamol on proliferation, apoptosis, and necrosis in the pancreatic 
cancer cell lines PaTu 8988 t and Panc-1. BMC Pharmacol Toxicol 18(1): 77.

30. Bundscherer AC, Malsy M, Gruber MA, Graf BM, Sinner B (2018) Acetaminophen and metamizole induce apoptosis in HT 29 and SW 480 colon carcinoma cell lines in vitro. Anticancer Res 38(2): 745-751.
31. Velasco G, Hernández-Tiedra S, Dávila D, Lorente M (2016) The use of cannabinoids as anticancer agents. Prog Neuropsychopharmacol Biol Psychiatry 4(64): 259-266.

32. Kis B, Ifrim FC, Buda V, Avram S, Pavel IZ, et al. (2019) Cannabidiol-from plant to human body: A promising bioactive molecule with multi-target effects in cancer. Int J Mol Sci 20(23): E5905.

For possible submissions Click below:

\author{
Submit Article
}

